\title{
Imaging Techniques in Brain Tumor
}

\author{
Mingkun Yu, Wei Ye, Xiangqian Qi, Jun Qian, \\ Zhenyang Liu and Wenfeng Fang \\ Department of Neurosurgery, Changzheng Hospital, \\ Second Military Medical University, Shanghai \\ China
}

\section{Introduction}

Recent years, the emerging new imaging techniques not only show the detailed and complete aspects of brain neoplasm, but also promote clinical doctors using new techniques, such as CT three-dimensional reconstruction, magnetic resonance spectroscopy (MRS), diffusion weighted imaging (DWI), diffusion tensor imaging (DTI) to research the mechanism of those diseases in the aim of better treatment. The X-ray is the fundamental technology in clinical medicine. As CT and MRI make a great progress, the X-ray takes smaller and smaller part. When the neoplasm causes skull destruction or erosion, such as osteoma or Ewing's sarcoma of skull and pineal gland calcification, a significant X-ray can give us some useful information in diagnosis. The application of CT new technology, such as electron beam CT, dynamic CT enhancement, CT angiography and CT perfusion enhancement unfold a new boundary to neurosurgeons. Dynamic CT can not only reveal the pathophysiological change, but also make us keep an eye on local blood flow alteration. The CT angiography contains 4 methods which are threshold shaded surface display (SSD), maximum intensity projection (MIP), curved planner reformation (CPR) and volume rendering (VR). In those methods, three-dimension reconstruction takes the most important place, which gives us comprehensive observation by the complex show. On the other hand, if the blood vessels of tumor tissue need to be showed, the CT perfusion enhancement is a not bad choice, which can give clinical doctors accurate risk evaluation. The location, structure and perfusion of the neoplasm are the most important, and the answers of those significant aspects could be offered by conventional MRI. According to the distribution of the blood vessels, brain tumors can be divided into 3 types, and those provide a better differential diagnosis. Time-of-flight (TOF) and phase-contrast (PC) are the basic image formation methods. Multiple overlapping thin-slab acquisition (MOTSA) is the updated technology, which can elevate the signal overlapped vessels, display the relationships between vessels and brain. MRS can show the fineness and further, the metabolic characters of intracranial tumor. The MRS is complementary in brain neoplasm diagnosis, differential diagnosis, grading, evaluating and recurrence predicting. By determining the micrometabolite such as $\mathrm{N}$-acetyl-aspartate (NAA), creatinine ( $\mathrm{Cr}$ ), choline (Cho) etc in brain, MRS could be generally applied to the brain tumor. NAA is generally accepted as a marker in central nervous system. It decreases in almost all malignant brain tumors. The Cho extremely increases in malignant brain tumors, in the meaning that conversion function of 
cellular membrane is greatly accelerated. In most cases of malignant brain tumors, the $\mathrm{Cr}$ often lays on a lower level, compared to the normal value. With the use of MRS, the localization methods should be mentioned, which contain depth-resolved surface coil spectroscopy (DRESS), pointed- resolved surface coil spectroscopy (PRESS), spatially resolved spectroscopy (SPARS), the stimulated-echo acquisition method (STEAM), image selected in vivo spectroscopy (ISIS), fast-rotating spectroscopy (FROGS), point-resolved rotating-gradient surface-coil spectroscopy (PROGRESS) and volume-selective excitation (VSE). The MRS can be used to classify glioma, meningioma, metastatic tumor and other intracranial neoplasm by special peak and some characters. So, the MRS can tell us the characters and growth potential of a certain kind of brain tumor, and help us choose a correct and appropriate treatment strategy. Functional MRI is used to show the changes in the ratio of intravascular oxyhemoglobin to deoxyhemoglobin in response to a stimulus. It is widely used in many fields, such as vision, auditory perception, sensation, motor etc. which can be an addition to conventional MRI by discriminating neoplasm and edema. The functional MRI will be a guide in neurosurgery surgical approach to decrease the mortality in preoperative period. The diffusion of the water in central nervous system is depended on the integrity of the cell membrane and the osmotic balance. Though each brain tumor has its own special DWI, which can supply some valuable information of diagnosis and differential diagnosis, but DWI is mainly used in brain ischemic processes. The diffusion of water first decreases due to cell swelling, in the reason of decreasing the extracellular space. During the recovery stage, the loss of cell wall causes the diffusion to increase. In MRI, fiber bundles' integrity, compactability and parallelism are described by fractional anisotropy (FA). DTI is the conversion of FA value to FA figure. FA figures can distinguish grey matter and white matter, disclose the diffusion character of brain and the details as internal capsule, corpus callosum which could not be seen clearly on conventional MRI. Another echo planar imaging (EPI) is used to measure tissue perfusion. As diffusion, its effect can be easily swamped by patient's minor motion. The method of imaging tissue perfusion is to create an endogenous contrast by presaturating the blood flowing into the brain tumor in a reasonable radio frequency pulse. So, the ever developing world will provide us more and more useful image techniques, and now let's go into details of the summary we talked above.

\section{X-RAY and brain tumor}

Since 1895, Roentgen discovered $\mathrm{x}$-ray which was used as a traditional $\mathrm{x}$-ray machine till to 1970s. The x-ray machine brought about a structural change and improved its qualitative leap in performance in 1980s. In the late 1990's, CT and x-ray technology were combined to produce a new generation of digital x-ray (DR) radiographic techniques. So far, DR technology is widely used in clinic. X-ray diagnosis of brain tumors is mainly made by indirect signs. Skull destruction or hyperplasia or cranial calcification showed on $\mathrm{x}$-ray, can be used for rough diagnosis of brain tumor. As X-ray technology is relatively simple and low cost, it is important in the initial diagnosis of brain lesions for the people living in some remote areas or underdeveloped regions. DR powerful quality control module of the postprocessing techniques ensures the stability of image quality and avoids image distortion caused by scattering, blur and distortion. But the DR image as ordinary x-ray image, twodimensional image is not only lack of three-dimensional effect, but also to be strict in the design requirements of the body. DR images are more sensitive to respiratory and body 
movements。In the diagnosis of brain tumors, x-ray technology is far lower than CT, MR imaging technology. Plain film can sometimes show some signs which suggest the location of the intracranial space-occupying lesions according to the site of tumor calcification. Lesions could occupy some space within the cranial cavity, which are bound to cause some intracranial structural movement. Lesions near the skull may cause local bone destruction or proliferation often due to tumor oppression or erosion. These changes with positioning significance, directly or indirectly suggest the location of lesions. Tumor calcification results from tumor necrosis or hemorrhage, and often locates in the tumor and its vascular wall. Therefore, the appearance of calcification may specify the location of the tumor. Calcification ranges from a few millimeters to several centimeters, but the size of calcification can not represent the size of the tumor region. The site of tumor calcification is confined to the cranial cavity, which may be deep in the cranial cavity (such as glioma, etc.). Tumor calcification form may be irregular, nodular, patchy or small point, and wall calcification more features, which often has loop-shaped outline form cysts. If the calcification occurs and develops slowly, it mostly looks like benign calcification. The incidence of calcified craniopharyngioma ranks first in intracranial tumors in children. Most of intrasellar calcification is close to the saddle diaphragm and the minority may locate in the saddle back. The most common sign is the small number of high-density spots, or tiny piles of small pieces together. These spots or small pieces of calcified wall of the tumor spread fairly frequently so that the location can be another form of projection such as the arc form whose dense wall calcification is prone to shape and even depicts the outline of the entire tumor. The number and extent of calcification vary in size and sparsely scattered mostly from 1 to several centimeters in diameter. Large calcification is rare. Sometimes only one or two small point's calcification occurs, but the tumor is very large. Calcification of meningioma may be seen only in sand type and curing specific form. Calcification of meningioma generally occurs around $10 \%$, sometimes up to $20 \%-50 \%$. Most of calcification locates in the periphery of the cranial cavity, or the skull base close to the parietal bone surface. Differential diagnosis between Physiological intracranial calcification and calcified tumor are more difficult. CT and MRI should be further examined if there is difficult in diagnosis. The existence and the site of intracranial lesions could be inferred according to the displacement of the normal physiological intracranial calcification such as pineal calcification. Pineal calcification locates in the midline of cranial cavity. Cerebral hemisphere lesions can easily lead to its opposite displacement. Local bone changes include decreased density, bone destruction and bone hyperplasia due to the long-term oppression and erosion of the brain tumor. Lesions beside the sella may cause deformation, destruction or resorption of the ipsilateral sella bone structure. Invasive skull base tumor in the sella can cause destruction or resorption of cranial bone. Lesions near the skull base, can cause the expansion and deformation of the local canals. The pore size of the normal skull base is individually different, and both sides of them can also be slightly asymmetric, so diagnosis must be carefully considered. The expansion of the internal auditory canal is common in acoustic neuroma.

\section{The computer tomography of brain neoplasm}

As the popularity of the computer tomography (CT), it has been the first choice in diagnosing intracranial neoplasm. The X-ray getting through the collimator forms lots of electronic beam, and there is a detector bar in appropriate position in the aim of receiving 
the information of electron beam. After signal enhancement and analog/digital converter, the information will be processed by MATRIX, and the CT picture could be got. The highlighted character of $\mathrm{CT}$ is the discriminating ability such as showing brain structure. Therefore CT can not only show the white matter and grey matter, but also disclose internal capsule and basal ganglion. And the less loss of information can provide us more useful message to diagnose and evaluate prognosis. However, completely relying on $\mathrm{CT}$ in diagnosis and differential diagnosis, is not a good idea. Because the same tumor may present different picture and different neoplasm can show the same character, the application of different imaging method should be put in the first place, and this can give us clinical doctor lots of useful messages. Before CT scanning, some points should be talked about. First one is CT value and partial volume effect. The X-ray absorption in certain area can be evaluated by CT value which presents the density of normal and abnormal tissue in human. The partial volume effect will affect the result of CT picture. In case of neoplasm not displayed in all scanning pictures, CT value will show different density around the same tissue. The adjustment of window width can show the fine difference between normal tissue and neoplasm, and this is the second point should be noticed. The choice of window width and window center depends on the requirement of clinical doctor. Large window width leads to more structures presenting and less details showing. So the correct window width and window center is very important in CT scanning. And the third point is the enhancement. The picture will be enhanced when the neoplasm contains rich blood supply. This can give doctors better judgment in diagnosing and treatment. The last point is the artifact which come from different additional reasons, and in this situation, a re-examining should be in consideration. CT perfusion imaging is a new technology. Through this method, some parameter as blood flow, blood volume, mean transit time and time to peak could be acquired to evaluate the brain neoplasm. It is simple and feasible, and could be used in lots of area. After contrast enhancement, a time density curve could be got through multi-scanning to a certain area, and reconstructure of the data could provide us the perfusion situation of the organ. The growth period of brain tumor can be divided into two parts: first one without vessel and the second with vessel. In first period, the slow growth of tumor is in the short of nutrition supply, and then the formed vessels supply tumor rich nutrition so that the tumor grows at a higher speed. Pathologist Weidner N analyzed the tumor vessels and formed notion of microvascular density, which can give us information of the neoplasm to make appropriate treatment schedule. At present, microvascular density has become the "golden standard" in evaluation the growth of tumor vessels. But in the clinical process, the use of the criteria needs re-evaluating because of dynamic change reasons. Because different tumor has its own perfusion parameter (Wintermark et al; 2001), CT perfusion imaging could be used for diagnosis of brain tumor. The CBV of high grade glioma is quite different from that of the low one. And the more blood supply, the higher the $\mathrm{CBV}$ and $\mathrm{CBF}$ value will be detected. Recent research shows that the $\mathrm{CBF}$ value increase significantly in glioma and meningioma. Conventional CT and MRI cannot outline the tumor for infiltration, the reason of which lies in the integrity of blood vessel. While perfusion CT can give us the message of different parameter we want (Cenic et a1; 2000). In guiding biopsy, our clinical doctor could take the tissue from the higher perfusion area, and elevate the successful chance of biopsy, promote us make the very suitable plan for patient treatment. The perfusion parameter has close relationship with the blood supply of 
neoplasm (Natbavi et a1; 2001). Use this technology, the necrosis after radiology and recurrence can be distinguished. If CBF ratio of the enhanced area to the normal brain higher than 2.6, the tumor recurrence is in consideration, while the ratio lower than 0.6 , a tendency to be necrosis should be thought over.

The principle of dynamic CT is that time density curve will be obtained through processing a series of scanning after enhancement. Different tumor has different time density curve. The curve could be divide into five kinds: first, fast perfusion and diminishing with high peak, which can be seen in vascular disease; second, slow perfusion and diminishing with no so high peak, whose curve is mostly seen in meningioma, pituitary adenoma and metastasis tumor; third, slow perfusion and diminishing with low peak, which can be seen in glioma; fourth, slow perfusion and fast diminishing with low peak and the 5th one, a linear form. As the dynamic CT can show the brain vessels very clearly, so it not only can distinguish brain tumor from the vascular disease, but also have some meanings in differential diagnosis of brain tumor. Dynamic CT can help us understand the enhancement process in a convenient way. Before the peak, the data mean the perfusion of tumor and after the peak, the data show the resident of enhancer. There are four stages in dynamic CT time-density curve, slow increase of density, fast increase of density, fast decrease of density and slow decrease of density. The average time should be from 12 to 19 seconds. The peak can distinguish tumors rich or short of blood supply.

\section{MRI and brain tumors}

Magnetic resonance imaging has been an important component in the medical imaging diagnostic field. The research showed that MRI for intracranial tumor is better than CT, whose accuracy reaches $98 \%$ in positioning diagnosis and $70 \%-85 \%$ in qualitative diagnosis. MRI has the following advantages: First, MRI has better soft tissue contrast; Second, 3-D imaging is more advantageous to accurate orientation of the lesions by multiple parameters and directions of the organs; Third, MRI can show intracranial vascular structure without contrast; Forth, No radiation damage, which is safe and reliable. In recent years, new technology of magnetic resonance imaging including DWI, DTI, PWI, fMRI and MRS, which have been in clinical research and application, is developing quickly. It not only reflects the information of the anatomy changes, but also provides the information of pathology, physiological function and biochemistry. So more accurate positioning of brain tumor and more reasonable design of surgery have been made in pre-operation or operation, which can reduce the damage of brain function and neural networks effectively. The advanced technology of MRI has been widely applied.

\subsection{Conventional MRI and brain tumor}

At present, the 1.5 to 3.0 tesla MRI have been used in most hospitals. The usual screening MRI of the brain begins with a sagittal localizer of the brain. The other includes T1 and T2 weighted axial images and FLAIR sequence. In some centers, gadolinium is routinely injected for a contrast enhancement picture so as to have a better diagnosis.

Gliomas are the most common primary brain tumors in both adult and children, taking approximate $1 / 2$ to $2 / 3$ parts of all brain tumors. Gliomas are usually divided into four distinct histologic subgroups: astrocytomas, oligodendrogliomas, ependymomas and choroid plexus tumors. The appearances of gliomas in MRI can be quite different. In some 
cases, the neoplasms have a distinct location and performance, which lead into correct diagnosis. While in other cases, the MRI image is not specific, and the clinical diagnosis should be made after pathologic diagnosis. MRI appearances are also depended on the histological grade of the neoplasm. In other words, the low-grade tumors usually exhibiting minimal or no peritumoral vasogenic edema, and high-grade tumors show prominent vasogenic edema. However, the sensitivity and specificity of MRI are limited in assessing the degree of anaplasia neoplasm. Astrocytoma accounts for $20 \%$ to $30 \%$ of all gliomas. The histological subtypes such as fibrillary, protoplasmic and gemistocytic varieties, are hardly distinguished by MRI. Some other types, such as cystic pilocytic astrocytomas, are typically found in the cerebellum in children, and subependymal giant cell astrocytomas with tuberous sclerosis can be distinguished by MRI based on their typical appearance, location and clinical manifestation. On MRI T1-weighted images, astrocytomas usually present hypointense to normal, while on T2-weighted and FLAIR images, astrocytomas present hyperintense to normal. Sometimes the MRI pictures cannot completely reliable, but the general rules should be noted. Low-grade astrocytoma shows slight peritumoral edema and homogeneous in signal characters on both T1 and T2-weighted images, while high-grade astrocytomas are usually more infiltrative without defined borders, associated with significant surrounding edema and more likely to be heterogeneous with the cystic and necrotic changes typically found in those tumors. Enhancement of astrocytomas on postgadolinium T1-weighted images can be quite variable. Although high grade tumors generally demonstrate more prominent enhancement than low grade tumors do, exceptions often occur. So the presence or absence of significant enhancement should not be used to estimate the degree of tumor which might be certificated by pathology. Post-contrast T1weighted images can improve the sensitivity of MRI in the preoperative diagnosis and postoperative recurrence of those neoplasms. This sensitivity can be further improved by MR spectroscopy, which shows significantly elevated choline amplitude rated to creatine would suggest a recurrent form. The absence of elevated choline in a background of mobile lipids and lactate would favor radiation necrosis or post-surgical changes. New MRI techniques such as perfusion MRI and functional MRI are now more and more used. The MRI of oligodendroglioma shows more calcification, and usually hypointense on T1 and T2weighted image, but microcalcifications can present hyperintense on T1-weighted images. So it is not very reliable by MRI to differentiate those tumors. The ependymomas have a protean MRI appearance and variable signal intensities on T1 and T2-weighted images, which are consistent with the variable cellularity and histologic composition of tumors. They are typically hypointense on T1-weighted images and hyperintense on T2-weighted images. However, calcification and cystic or hemorrhagic components often result in a variable, heterogeneous MR signal and enhancement.

Meningiomas are the second most common primary brain tumors. They are usually found in middle-aged to older women. As to pathology, they arise from the arachnoid cap cells and are commonly found over the parasagittal cerebral convexity, sphenoid wing, parasellar, tuberculum sella, olfactory groove, and cerebellopontine angle regions. They are usually isointense to grey matter on both T1 and T2-weighted images and therefore can be overlooked on screening MRI studies without contrast. They are often associated with an enhanced, thickened dura along the lateral margins of the tumor, which is known as a "dural tail". Meningiomas sometimes have areas of necrosis, calcification, hemorrhage and cystic changes, which generally lead to relatively small amount of edema. 
Pituitary adenoma is one of the more common neoplasms encountered in adult neurosurgical patients. The tumor may be functional or non-functional adenoma. The functional adenoma can secrete prolactin, adrenocorticotropic hormone, growth hormone and so on. But MRI can not distinguish those subtypes. However, MRI is valuable in identifying a microadenoma. In case of macroadenoma, MRI can accurately identify the sellar, suprasellar, and parasellar extension of the tumor and the relationship between tumor and surrounding structures such as optic chiasm, optic nerves, hypothalamus, and cavernous sinus and supraclinoid segments of internal carotid arteries. The most important imaging sequence is the T1-weighted images with gadolinium contrast, which should include thin coronal images through sella turcica with a small field of view and filmed with magnification. In microadenomas, dynamic images should be done with a series of thin (3 to $4 \mathrm{~mm}$ ) coronal images rapidly obtained over 2 to 3 minutes during bolus intravenous injection of gadolinium. It can identify a relatively slow enhancing microadenoma, within a rapidly and homogeneously enhancing normal pituitary parenchyma. Some other image findings include a deviation of the infundibulum away from the centre of the gland containing the adenoma, asymmetrical convexity of the superior border of the gland, and abnormal contour of the floor of the sella turcica. In the evaluation of macroadenomas, additional imaging sequence including T1-weighted sagittal images with contrast and T2weighted coronal images, are valuable in defining tumor extension and associated parenchymal changes.

MRI with gadolinium contrast is the most sensitive imaging technique for the evaluation of the central nervous system metastasis. For the reason of multiplanar evaluation and no CT artifacts, MRI is superior to CT in tissue contrast. The appearance of metastasis tumor depends on the original tissue. The lesions are typically localized at grey matter-white matter junction of the supratentorial brain and occur more commonly in the anterior circulation. On T1-weighted images, the lesion often show iso- to hypo- intense, while on T2-weighted images, the lesion often present iso- to hyper- intense with prominent surrounding vasogenic edema.

\subsection{Contrast-enhanced MRI and brain tumor}

The MRI of enhanced T1-weighted images has great values of orientation, qualitative assay and curative effect evaluation in intracranial neoplasm (Zimmerman et al., 1999). It also exists some certain limitations, such as the difficulty for showing lesions in ventricle side or cortex area, not accurately reflecting the blood, the actual size and the scope by strengthening region and not offering the information of function in tumor. Currently the new technologies of MRI including contrast enhanced FLAIR MRI, dynamic contrast enhanced MRI, perfusion weighted MRI can provide more information for clinical treatment, which compensated for the shortage of conventional enhanced MRI. FLAIR is a stronger T2-weighted image by extremely long TR, TE and TI around 2000ms. The characteristic of image is that free water signal is zero and the contrast of brain grey matter is low. But the contrast between the lesion and the background is significantly increased in T2-weighted images. So the lesion is showed more clearly due to eliminating the influence of cerebrospinal fluid in ventricle side or brain ditch (Husstedt et al., 2000; Singh et a1., 2000). The research (Mathews et al., 2000) showed that enhanced FLAIR not only can express enhanced T1-weighted images but also can better detect the lesions of superficial parts compared with it. Enhanced FLAIR images which can observe the strengthened lesions and the edema area, makes up for the shortage of T1-weighted and T2-weighted 
images in some degree (Fig.4-1). Some scholars (Ercan et a1., 2004) suggest that enhanced FLAIR images should be used as conventional scan and then the T1-weighted and T2weighted images abolished, especially in detection of brain metastases and following-up for treatment of brain tumor.

The basic principle of dynamic enhanced MRI is the T1-weighted relaxation effect and magnetic sensitive effect of organizations caused by paramagnetic contrast agents through vein injection. Smaller doses of contrast agents can obtain higher resolution images. After several scans continuously, the change of strengthening degree in organization can be observed dynamically, and the hemodynamic quantitative material also can be obtained (Wong et al. , 1998). Through observing and analyzing the parameters of the interesting area such as SI-T curve, SImax, Tmax, Va and MCER, the information of differential diagnosis, the type and the grade of tumor can be obtained. Because it has the characteristics of no magnetic sensitive artifact and little effect by the surrounding edema, the basal ganglia lesions can be displayed obviously. PWI is the images of using dynamic contrast through magnetic sensitivity. It can obtain hemodynamic materials that MRI and MRA can't get such as $\mathrm{rCBV}, \mathrm{rCBF}$, and rMTT. It also can get the information of neural function and cerebrovascular state. So PWI can be used to identify primary or metastatic tumor, benign or malignant tumor, recurrence or the necrosis after radiation therapy (Wetzel et al. , 2002). Because it has obvious magnetic sensitive border artifact in the bone-brain or gas-brain interface, PWI currently only applies to hemisphere lesions which is still have some limitations in skull base or raised-face lesions.

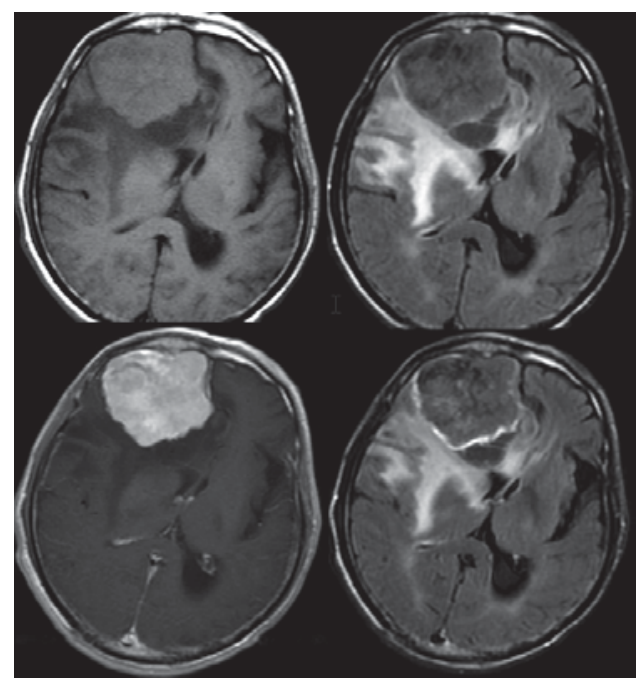

Fig. 4-1. T1WI (upper left), FLAIR (upper right), enhanced T1WI (lower left); contrast enhanced FLAIR (lower right).

\subsection{DWI and brain tumor}

DWI reflects the Brownian motion of tissue water. The diffusion of water molecules is faster, the signal is lower. The slower part of diffusion shows high signal. In ADC images, the organization with faster diffusion has high ADC values, and ADC value is low in the 
organization with lower diffusion. It can be used to diagnosis or differential diagnosis in intracranial neoplasm. It also not only can provide the clinical basis of rational operation plan, but also can be a method for monitoring the results after healing, which makes up for the shortage of conventional MRI.

\subsubsection{DWI in diagnosis and differential diagnosis}

Intracranial cystic lesions mainly include epidermoid cyst, dermoid cyst and arachnoid cysts. In SE sequences, there are long T1, T2 signal and no strengthening with enhancement. In DWI, epidermoid cyst and dermoid cyst are showed high signal and low ADC values, but arachnoid cyst is expressed low signal and high ADC values (Reddy et al., 2006). Common intracranial lesions including cerebral abscess, glioma and metastasis with hoop strengthening in MRI traditional sequence, have the same performance whose diagnosis is difficult. There are a variety of inflammatory cells, bacteria, necrotic tissue and sticky liquid secretion in the brain abscess in which the diffusion speed of water molecules is significantly limited. So the ADC values drop obviously, and DWI has high signal. But the cystic changes or necrotic area of brain tumor only include necrotic cells, bits of inflammatory cells and serums. Its ADC values and DWI signal are similar to CSF (Mishra et al., 2004).

In conventional MRI images, it is difficult to distinguish between the central nervous system lymphoma and gliomas before the operation. Because lymphoma cells density is higher, the ADC value is often lower than that of gliomas (Guo et al. , 2002) (Fig.4-2).

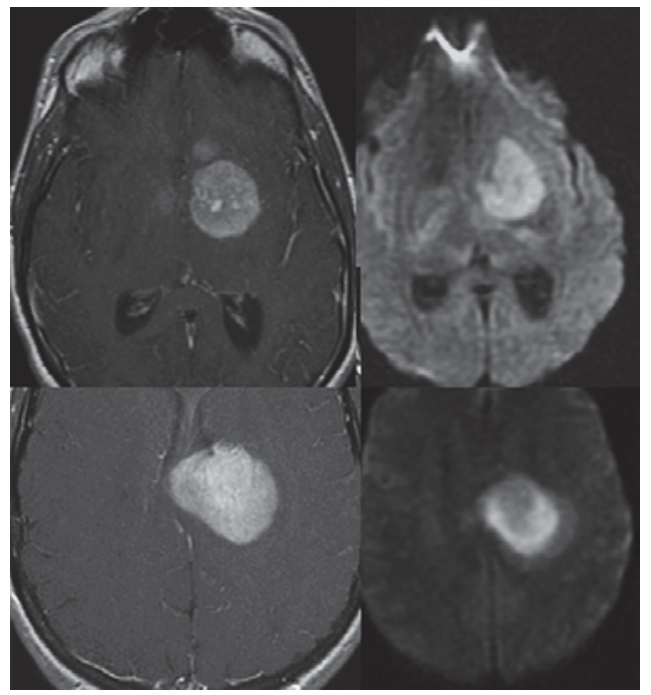

Fig. 4-2. T1 with contrast (left), DWI (right), primary central nervous system lymphoma (top row), GBM (bottom row).

\subsubsection{Preoperative assessment of surgical resection range}

DWI has certain effect in judging the low grade glioma with no strengthening and the malignant tumor boundaries which invade surrounding tissues. The edema area around glioma can be found not only the capillaries and edema, but also the invasive growth of tumor 
cells scattering along the new blood vessels or expand blood vessels especially in high grade glioma. Because of the inverse relationship between ADC values and cell density (Hayashida et al. , 2006), the ADC value of edema area around malignant glioma is relatively lower. It is very important to determine the range of surgical resection in preoperation.

\subsubsection{Monitoring the effect of therapeutic response}

ADC images which have potential value for evaluating the efficacy of fixed-point treatment such as radiation and local intracerebral injection of chemotherapy drug can be used to reflect the local treatment response quantitatively. The research (Hamstra et al., 2004) showed that ADC value before treatment is negatively correlated with extinctive degree of tumor after treatment. ADC level of tumor has been regarded as a imaging indicator of forecasting therapeutic effect. Another research (Morat et al. 2005) also confirmed fDM in the treatment period can predict the response to treatment correctly in the 20 patients which was calculated the correlation between tumor diffusion value and clinical response by DWI imaging before radiotherapy or chemotherapy and after three weeks, and regarded the dispersion value of water molecules in tumor as an early markers before tumor volume change.

\subsection{DTI and brain tumor}

In recent years, DTI has been rapidly developed a new MRI imaging technology based on weighted imaging. It is currently the only alive, noninvasive and individualized 3D imaging technique which can display the fiber structure of cerebral white matter (Poupon et al. 2000) (Fig. 4-3). DTI is similar to DWI but involves the collection of additional data

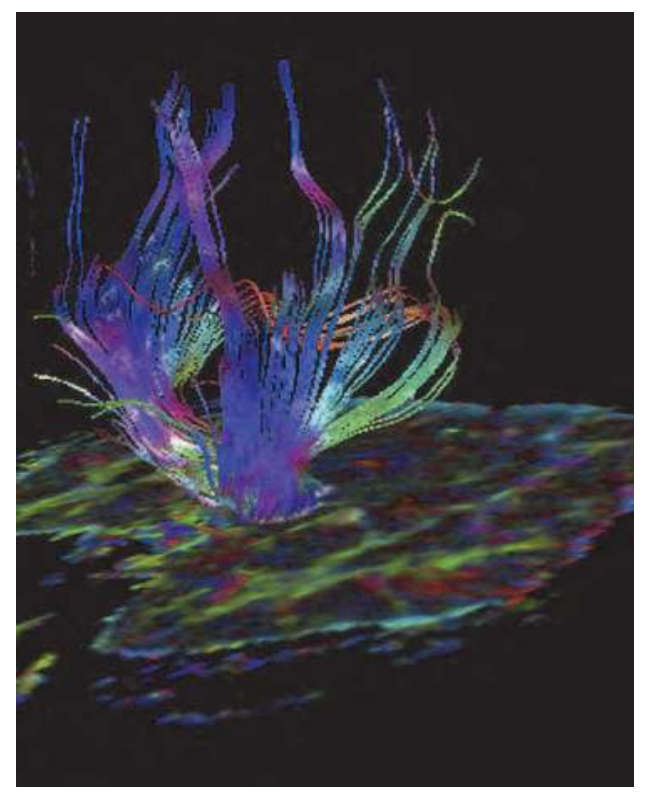

Fig. 4-3. Tractogram from a third subject illustrates the standard technique for displaying DTI data for surgical planning. (tractogram images courtesy of Kelvin Wong, PhD, using Philips Pride Software at Jockey Club MRI Center, University of Hong Kong.) 
necessary to define the tensor describing the preferential direction and magnitude of water diffusion. The degree to which water diffusion in tissue is facilitated in one direction and hindered in another-referred to as diffusion anisotropy is often characterized by a scalar value derived from the diffusion tensor: fractional anisotropy.

\subsubsection{DTI in diagnosis and differential diagnosis}

Compared with metastases, the FA value is significantly lower in glioblastoma (Wang et al., 2009). However, the FA value is higher and the MD value is lower around glioblastoma (Lu et al., 2004). The FA, ADC value and the ratio of them in lymphoma is obviously lower than in glioblastoma (Toh et al., 2008). Compared with meningioma, the FA value around the area of tumor is lower in glioma (Provenzale et al., 2004). The average FA value of edema surrounding metastatic and its ratio is lower than in meningioma, but the MD values and its ratio are higher (Toh et al., 2007). The FA value in DTI is controversial in classifying the gliomas (Inoue et al., 2005; Goebell et al., 2006; Lee et al., 2008). The main reason of inconsistent results is hard to limit interested standard regions for effective data analysis.

\subsubsection{DTI in treatment}

Because DTI can clearly show the relationship between white matter fibers and brain tumor, it has great clinical value for guiding surgery including preoperative plan, intraoperative navigation and postoperative evaluation. The relationship between nerve fibers and brain tumor can be divided into three kinds: damage, shift and infiltration. The results showed that meningioma, glioblastoma and astrocytomas make the fiber bundles shifted, destroyed and infiltrated respectively (Wei et al., 2007). As a result, we can formulate operation plan and decide to surgical approach through confirming the relationship between white matter fiber bundles and brain tumor in preoperation. Through combined intraoperative DTI with fMRI and navigation technology to locate the positions of fiber bundles, brain function and brain tumor, it can be used as guiding tumor resection in intraoperation. At the same time, the maximum range of tumor is resected with the result that the important functional areas and fiber bundles are preserved greatly (Talos et al., 2006). DTI can also be applied to observe the therapeutic effect of drugs on tumor by dynamic tracking display in addition to the comparison of integrity and position of fiber bundles in postoperative evaluation (Wu et al., 2007; Gerstner et al., 2008). Meanwhile, the FA value is also a useful biological index of nerve damage caused by the treatment (Khong et al., 2006).

\subsection{Bold-fMRI and brain tumor}

BOLD-fMRI image is got by using endogenous hemoglobin as contrast agents through blood oxygen saturation alteration which reflects the changes of blood flow, blood volume and hemoglobin oxygen cooperation at neural activity. The different signal changes of cerebral blood oxygen consumption between activated area and not activated area are statistically analyzed to identify functional area.

\subsubsection{The application of fMRI in surgery}

The relationship and changes between lesions and functional areas in patients with brain tumor inspected by fMRI in preoperation contribute to design operation plan precisely. It can significantly reduce the risk of postoperative dysfunction. So fMRI should be the routine preoperative inspection in patients with brain tumor (Tomczak et al., 2000; Haberg et al., 
2004). There may be an adapted response of functional areas after injury because of mass effect by tumor which makes the cortex functional area deformation, shift and restructuring. Combined with intraoperative behavior evaluation, fMRI can protect the patients' cognitive function in intraoperation (Amiez et al., 2008). Understanding the relationship between cortical activated area and tumor in preoperation, it may help us predict the dysfunction risk degree and the possibility of tumor recurrence after tumor resection (Wildfǒrster et al., 1998).

\subsubsection{The combined application of $\mathrm{FMRI}$ and other methods}

4.5.2.1 The combined application of fMRI and neural navigation

Intraoperative navigation which is conducted through the anatomical and functional orientation logged in stereotactic neurosurgery navigators by $\mathrm{fMRl}$ in preoperation can effectively remedy the defects of intraoperative orientation, avoid intraoperative injury of cortical functional areas, reduce postoperative complications and lower the recurrence rate after surgery (Hall et al., 2009).

\subsubsection{The combined application of $\mathrm{fMRI}$ and DTI}

The combined images of fMRI and DTI in preoperation can show not only the cerebral cortex function, but also the brain white matter fiber bundles. Its value is higher than fMRI alone which make less postoperative complications and help to improve the prognosis because of the good assessment of function in language, movement and vision (Ulmer et al., 2004) (Fig.4-4).

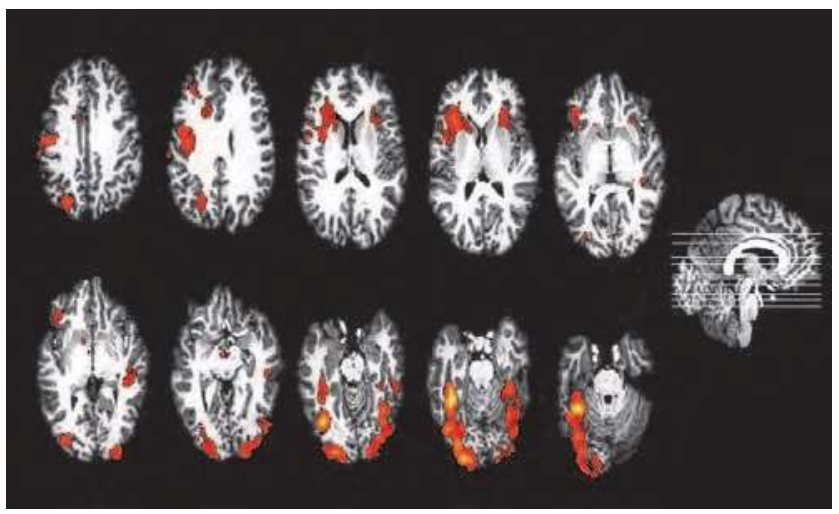

Fig. 4-4. fMRI results to word reading.

4.5.2.3 The combined application of fMRI and 3D-ultrasound

The data of fMRI and DTI can be integrated into 3D-ultrasound in order to correct the deviation caused by brain shift (Rasmussen et al., 2007).

\subsection{MRS and brain tumor}

As the MRS was admitted to the clinical application in 1995, neuroradiology has step into a brand new world. Being a non-invasive examination method, MRS can provide us the metabolic information which the conventional MRI and CT cannot tell us, and the one more important aspect, the MRS can not only find some abnormal earlier than conventional MRI, 
but also provide the prognosis information. Each compound in brain obtains its own picture in different frequency, and so it can be detected for several reasons: made of hydrogen, concentration more than $0.5 \mathrm{mmol} / \mathrm{L}$, own different frequency on chemical shift. As to the atoms used in the MRS, all of us will place the hydrogen in the first place. MRS can provide a "biopsy on line" without any invasive operation (Buascher et al., 2000), and can evaluate metabolic information on brain tumor (Fountas et al., 2000). Middle-time-echo (144ms) shows the elevation of choline ideally, which is the major presentation in brain tumor. Shorttime-echo $(30 \mathrm{~ms}$ or $35 \mathrm{~ms}$ ) can detect $\mathrm{mI}$ to grade the neoplasm. The peak of NAA decreases because of the death of neuron, and the peak of $\mathrm{Cr}$ decreases in the reason of tumor metabolism. Cho is the most important chemical compound in evaluating the neoplasm, the increase of which often tells us the reproduction of the membrane. When the neoplasm present alike in the cMRI, MRS could be used for differential diagnosis. Cho is recognized as the special marker in brain tumor (Gruber et al., 2005). In high-grade (grade II and III) glioma (Negendan et al., 1996), MRS can show the high level of choline, but when the collecting of images in the necrosis area, the choline could diminish. So, trying to avoid necrosis area, is very important to presenting the Cho in glioma. In adult MRS, Lac peak could be detected and the high grade tumor often links with higher peak of Lac. And in meningiomas, increase of Glx can be detected(McKnight , 2004).

The accuracy of grading malignant brain tumor by MRS is higher than aimless biopsy. For two reasons: one is that MRS can analyze the relatively big amount tissue, and the other is when biopsy taken the part not in the active area could not provide the accurate grading of the tumor. The sensitivity, accuracy and specificity of discriminating high-grade tumor or low-grade tumor are $100 \%, 96 \%$ and $86 \%$ respectively. In assurance of tumor grading, the Cho, Lac, Lip, NAA, Cr and $\mathrm{mI}$ can provide us lots of useful information (Murphy et al., 2004). Within the high-grade tumors, the higher Cho, Lac, Lip and $\mathrm{mI}$ can be detected, but NAA and Cr often show a decreasing form. In the malignant brain tumors, because cMRI cannot show the exact boundary, MRS or PWI should be considered. MRS can also provide useful message on tumor progressing, the Cho increasing more than $45 \%$ often shows the progress of tumor. The cMRI and MRS has been used in neurosurgery operation in the aim of resecting the neoplasm in a great and safe way, which take more and more important part in neurosurgery (Hemfinghaus et al., 2002).

\section{Digital subtraction angiography}

Digital subtraction angiography (DSA) is a type of fluoroscopy technique used in interventional radiology to clearly visualize blood vessels in a bony or dense soft tissue environment. DSA eliminates the unnecessary organization image, leaving only the vascular image through digital processing. Its distinct image and high resolution provide a real threedimensional image for the position measurement, diagnosis and interventional treatment of vascular disease. DSA is mainly used for examination and treatment of systemic vascular diseases and cancer. Before the application of CT and MR, angiography was one of the most important methods for brain tumor imaging which display the displacement, stenosis and occlusion of normal blood vessels caused by tumor and determine the tumor size and location combined with tumor angiogenesis (tumor stain), then make a diagnosis. It is generally considered that the characteristics of a benign tumor are of uniform thickness, more radial of vascular morphology, homogeneous dense tumor staining and clear outline (Fig.5-1.). The developing time is similar with cerebral circulation because of more mature 
blood vessels. Contrarily, the characteristics of a malignant tumor are of different thickness, inhomogeneous distribution of rough edges of vascular and unclear light profile of tumor stain. Because of the immature vascular structures, veins often develop early as a result of the direct intersection of arteriovenous. With the development and wide application of CT and MR, angiography plays a declining role in the diagnosis of brain tumor. Now angiography is mainly used for the identification of cancer and vascular disease, preoperative evaluation and embolization of the intracranial tumors.

DSA can help us understand the blood supply and scope of brain tumors which is useful for interventional treatment and followed-up. For hypervascular intracranial tumors, especially meningioma and glomus jugulare tumor, preoprative angiography is mainly used for displaying tumors' blood supply and impact of important veins. Taking the meningioma for example, its blood supply is often divided into the following categories (Manelfe et al, 1973). ECA alone, ECA and ICA mainly by ECA, ECA and ICA mainly by ICA, ICA alone. For meningioma supplied both by ECA and ICA, the ICA often supplies the peripheral of tumor, while the ECA for the centre, most of which come from the ECA branch such as the dura artery. It's very important in the preoperative preparation for a parasinus meningioma to make sure whether sinus was completely obliterated (Caroli et al, 2006). Completely occluded dural sinus means a considerable formation of collateral circulation. For this type of tumor and related dural venous sinuses which has been blocked, completely removing them usually doesn't cause venous thrombosis. However, the occurrence of dural venous sinus obstruction after surgery is of high risk if the tumor has accumulated in the dural venous sinus without blocking, especially in the back of the superior sagittal sinus, transverse sinus and sigmoid sinus. The goal of preoperative embolization of intracranial meningiomas is to facilitate their surgical removal by reducing tumor vascularity and decreasing blood loss during surgery (Richter \& Schachenmayr, 1983; Manelfe et al., 1986; Engelhard, 2001; Hirohata et al, 2003). Preoperative embolization appeared to be very useful in large tumors with pure or predominant external carotid artery supply (convexity meningiomas), in skull-base meningiomas, and in middle fossa and paracavernous meningiomas. It was also useful in falx and parasagittal meningiomas receiving blood supply from the opposite side and in posterior fossa meningiomas (Manelfe et al., 1986). The external carotid artery embolization is often adopted for safety consideration. And the gelatin sponge or particles of 200-300 micron is often selected as material for embolization. The catheter should be as closer as possible to the vascularity of tumor when embolism begins (Fig.5-1). Embolization in tumor is better than that simply for feeding artery. The main complications of embolization include cerebral vasospasm, local fever and headache, skin necrosis may also occur. For there could be "dangerous anastomasis" or reflux, so the most serious complications are blindness, aphasia, and sensory-motor disorder as a result of embolization in the intracranial blood vessels. Therefore, the operation process of embolization should be slow and careful to prevent refluxing. DSA is also used for malignant brain tumor in arterial infusion chemotherapy. There has certain effect, fewer complications and less systemic adverse reactions when applying intracranial arterial infusion therapy in patients with brain metastases. For patients with brain metastases unsuitable for surgical treatment, arterial infusion chemotherapy is effective in improving the quality of life and prolonging the life.

Despite of the rapid development of CT and MRI, DSA technology is still the "gold standard" of diagnosis and treatment for vascular disease. Following the development of the 
computer technology, imaging equipment and contrast agents in the future, DSA technology will play an important role in diagnosis and treatment for brain tumor.

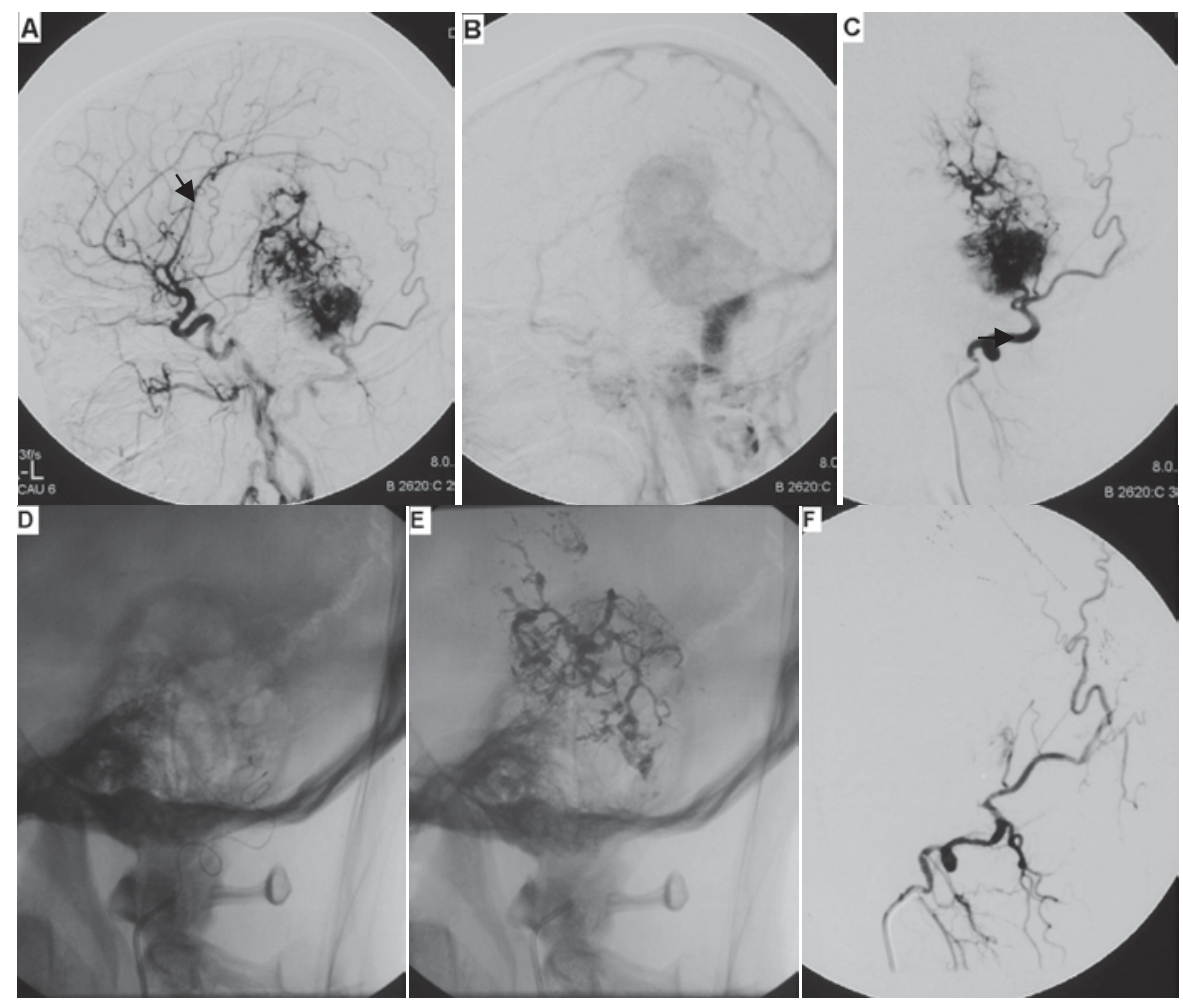

Fig. 5-1. Preoperative embolization for a meningioma. A, B, DSA displays the displacement of MCA (black arrow) and stain of the tumor. C, A super-selected angiography of the occipital artery shows the feeding artery (black arrow). D, Microcatheter in occipital artery arrived at the feeding artery. E, The emboliaztion agents in the tumor. F, Angiography of the occipital artery post-embolization.

\section{Perfusion imaging}

Perfusion imaging with CT or MRI is an exciting new radiological technique for noninvasive evaluation of cerebral hemodynamics in brain tumor. Cerebral perfusion imaging describes the passage of blood through the brain's vascular network. In this part, we will focus on the role of perfusion MR imaging in improving accurate diagnosis and monitoring brain tumors during therapy.

\subsection{The basic principle of CT perfusion imaging}

The theory foundation of CT perfusion imaging is nuclear medicine radioactive tracer dilution and center volume principles. The radioactive tracer is quickly injected into people's vein, which go to the target organ through the left ventricular. By the dynamic CT 
scanning for the selected slices, we can get the time-density curve (TDC) for each pixel in these slices. According to time-density curve, blood flow (BF), mean transit time (MTT) and blood volume (BV) could be calculated by using different mathematical models. This method can be used for evaluation of blood perfusion conditions in the tissue. Common perfusion parameters include reginal cerebral blood volume (rCBV) which means total blood volume in the specific brain area, and reginal cerebral blood flow ( $\mathrm{rCBF}$ ) which means blood volume that flows through particular brain regions in unit time. Their mathematical relationship is $\mathrm{rCBF}=\mathrm{rCBV} / \mathrm{MTT}$.

\subsection{The basic principle of MR perfusion imaging}

MR cerebral perfusion imaging shows blood condition of brain in the capillaries level by application of markers. The common parameters are blood flow, perfusion, and time and so on of brain tissue in unit volume. The main parameters include cerebral blood flow $(\mathrm{CBF})$,cerebral blood volume (CBV), mean transit time(MTT) that the contrast agents go through the tissue, and time to peak (TTP) of contrast agents concentration, etc. Gd-DT-PA is commonly used as external markers. Arterial water molecules are often used as endogenous markers. Arterial spin labeling is a new perfusion technique that does not require exogenous contrast; instead it exploits the spins of endogenous water protons that perfuse the imaging plane.

\subsection{Applications of perfusion Imaging}

Perfusion imaging, especially with MRI has become an integral component of the complete radiological assessment of brain tumors. Perfusion imaging may reflect angiogenesis status and hemodynamic change of brain tumor. There are two kinds of tumor blood vessels sources, one kind is tumorous neoangiogenesis of the host, and another kind is neovascularization of tumour itself. There is obvious relationship between biological behaviour and vascularization of brain tumor, whose characteristics are the damage of the blood brain barrier so as to increase vascular permeability. Therefore perfusion imaging can be used for the diagnosis and differential diagnosis of brain tumors. Because the flow of blood to the tumor increased significantly, regional cerebral blood volume (rCBV) value can reflect how much there are functional capillary quantity. The increase of $\mathrm{rCBV}$, quickly leaking into the surrounding tissue (contrast agents quickly enter extracellular clearance), can reflect tumorous biological activities. The more active tumor grows, the higher the malignant degree of tumor will be, and the greater $\mathrm{rCBV}$ value is, also the faster the risk of leakage. The new vessels are more tortuous than the native cerebral vessels, leading to increased mean transit times (MTT). Therefor, perfusion imaging is often useful to establish the diagnosis of tumor and to distinguish tumor from tumor mimics, for example, infective granulomas or tumefactive demyelination, which are hypoperfused with low values of CBV, CBF, and MTT. Perfusion computed tomography (PCT) allows to reliably classify gliomas and lymphomas based on quantitative measurements of CBV and KTrans (Schramm, et al. 2010). In comparison with normal parenchyma, low grade gliomas showed no significant difference of perfusion parameters, whereas high-grade gliomas demonstrated significantly higher values for KTrans, CBV and CBF. Lymphomas displayed significantly increased mean KTrans values compared with unaffected cerebral parenchyma but no elevation of CBV. High-grade gliomas show significant higher CBV values than lymphomas (Figure 6-1 and 6-2). MR cerebral perfusion imaging can also identify the benign or malignant brain 
tumors. In addition, rCBV of the brain tumor will significantly reduce after the chemotherapy, embolization or radiation therapy. MR perfusion imaging can also be used to identify with tumor recurrence or focal necrotic after treatment and evaluate prognosis or monitor the efficacy of treatment. Furthermore, numerous studies (Park et al., 2009; Schramm et al., 2010; Ludemann et al., 2009; Park et al., 2009) have shown that perfusion imaging have special superiority in noninvasively grading tumor histology preoperatively. Low-grade astrocytomas are hypoperfused as compared to grade III and IV lesions (Figure 6-3). Up to one-third of the high-grade tumors do not enhance on post contrast T1 weighted images, which may lead to a false radiological impression of low grade; unless a perfusion study is performed, which demonstrates hyperperfusion due to neoangiogenesis, which is associated with higher grade (Figure 6-4). Some authors (Law et al., 2002) have suggested that MR perfusion along with spectroscopy may aid in differentiating a high-grade primary tumor from solitary cerebral metastasis. Primary high-grade tumors are infiltrative by nature; hence the peritumoral edema shows elevated CBV values. In contrast, metastases are well encapsulated and noninfiltrative by nature and therefor the perilesional edema being purely vasogenic shows low CBV values. MR perfusion may also be used as a guide to direct stereotactic biopsies from the most aggressive component of a heterogeneous tumor. $\mathrm{Up}$ to one-third of high-grade tumors are under-reported at stereotactic biopsy and this may be because the biopsy has not been acquired from the most aggressive portion of the lesion.

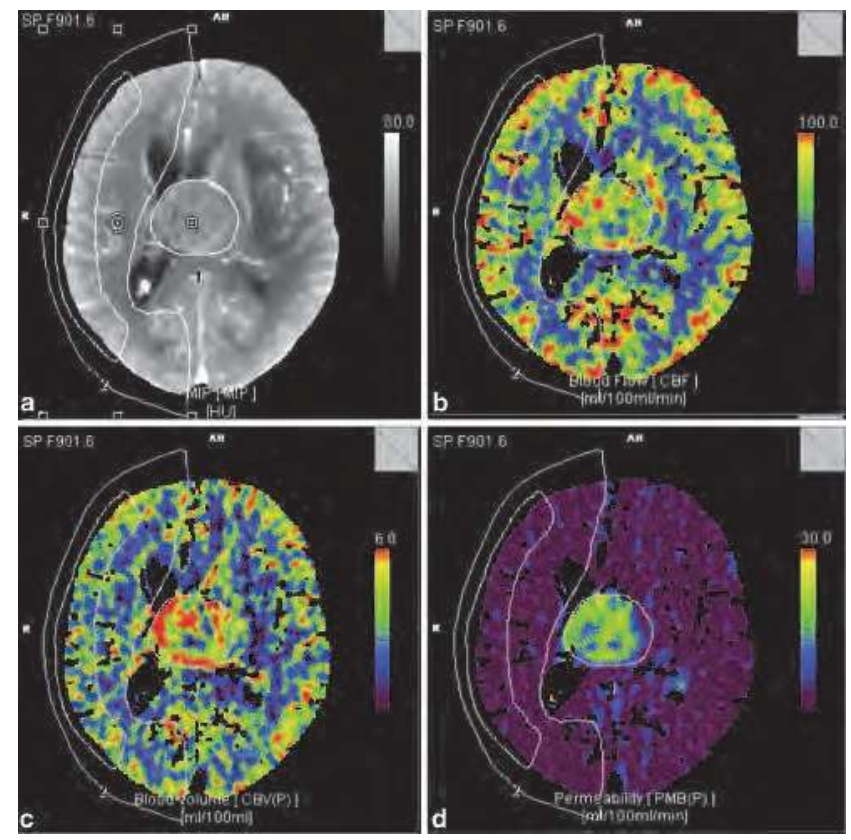

Fig. 6-1. A 58-year-old man with histopathological diagnosis of glioblastoma multiforme WHO IV. Axial contrast-enhanced MIP reconstruction image (a) shows a contrast-enhanced mass, which demonstrates intensely elevated blood flow (b) and volume (c) as well as strongly increased regional Ktrans (d) in comparison with the normal cortical and subcortical cerebral parenchyma (Schramm, et al. 2010). 

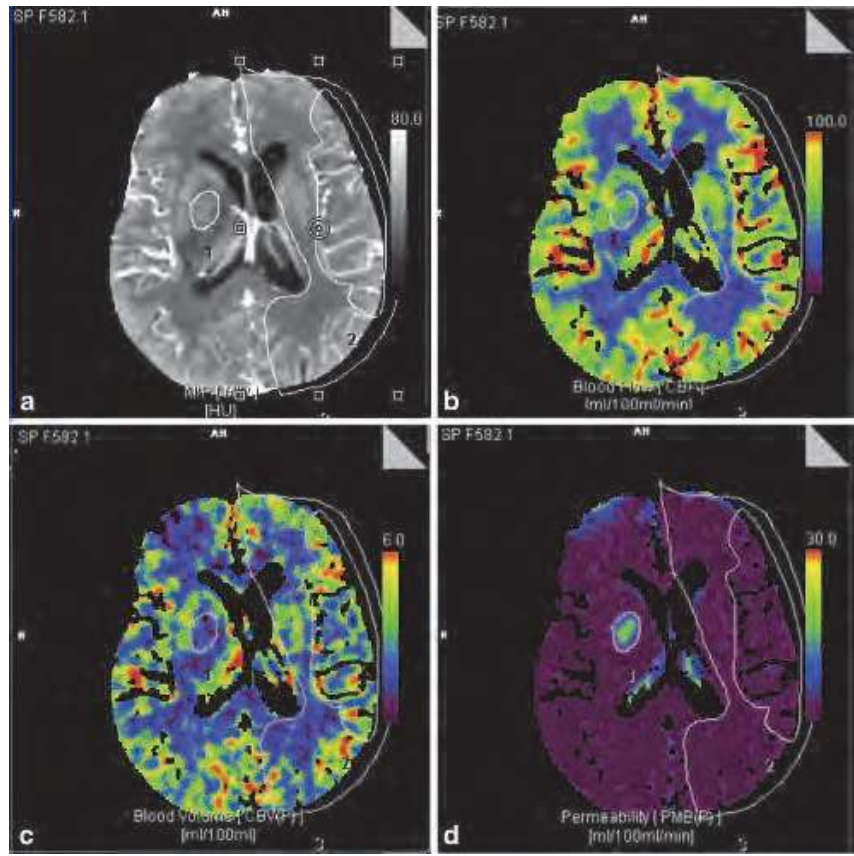

Fig. 6-2. A 72-year-old woman with histopathological diagnosis of primary cerebral lymphoma. In comparison with the contralateral normal cerebral parenchyma, the lesion depicted in the right lentiform nucleus demonstrates the typical perfusion characteristics of lymphoma: enhancement (a), no significant increase in CBF (b) or CBV values (c), though intensely increased regional permeability (KTrans, d), indicating a massive disturbance of the blood-brain barrier (Schramm, et al., 2010).
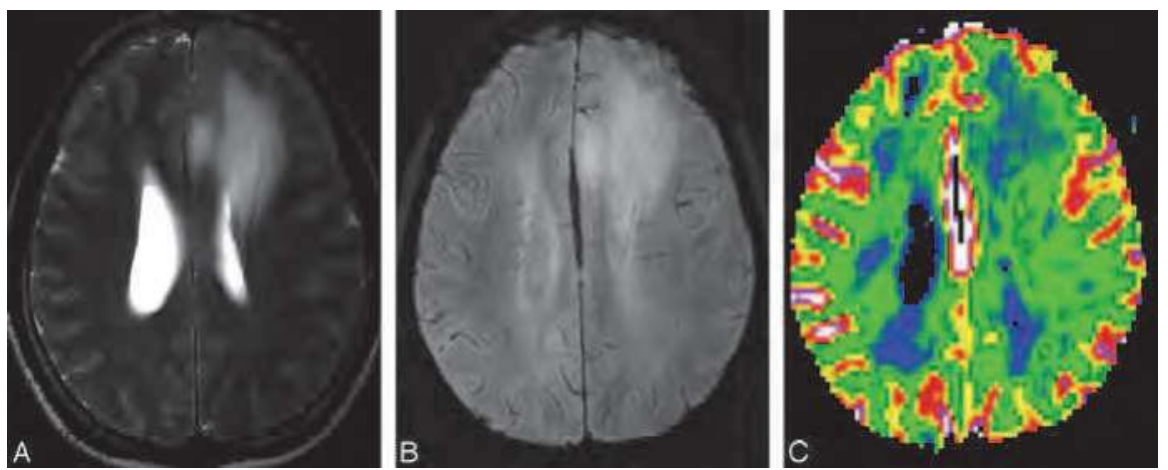

Fig. 6-3. MR images of a 39-year-old woman with left frontal low-grade astrocytoma (WHO grade II). A, The axial T2-weighted image shows an ill-defined mass with high signal intensity in the left frontal lobe. B, High-resolution susceptibility-weighted imaging (HRSWI) demonstrates no evidence of the intratumoral susceptibility signal intensity (ITSS). C, Corresponding rCBV map shows relatively low rCBVmax values of 1.31. (Park et al., 2009). 

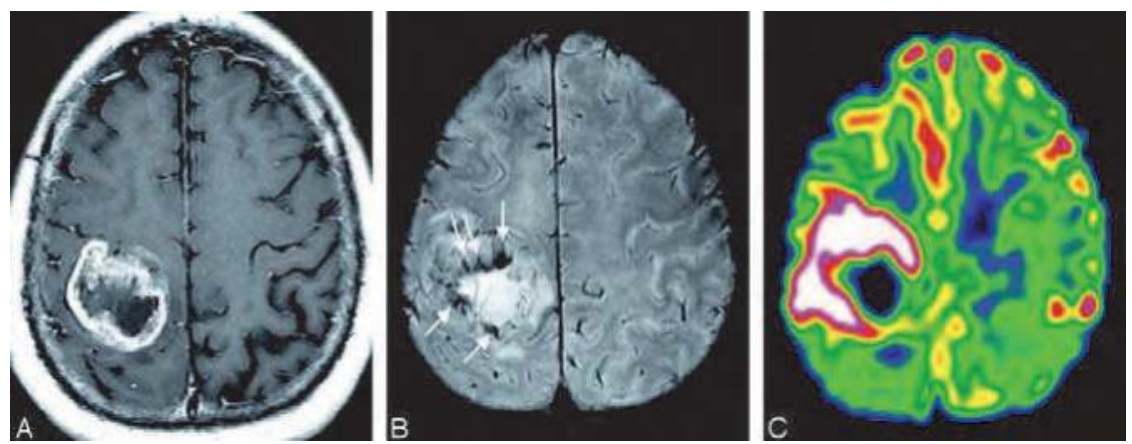

Fig. 6-4. MR images of a 55-year-old man with a glioblastoma multiforme. A, The contrastenhanced axial T1-weighted image shows a mass with irregular peripheral rim enhancement in the right parietal lobe. B, HR-SWI reveals conglomerated fine linear ITSSs just in the inner portion of the enhancing rim on the contrast-enhanced T1-weighted image (White arrows show tumor vessels). C, Corresponding rCBV map shows high rCBVmax values (4.71) in the tumor segment, including a maximum degree of ITSS. (Park et al., 2009).

MR perfusion can help distinguish gliomas from nonglial lesions like lymphoma and metastases or extra-axial tumors like meningioma. rCBV maps demonstrate increased blood volume in the enhancing high-grade glioma and decreased blood volume in lymphoma and metastases or extra-axial tumors, which are more significant in the area of peritumoral edema. This is because extra-axial tumors and nonglial lesions lack a blood brain barrier (BBB), hence, a very large fraction of the bolus leaks into the extravascular space during the first pass (Young G.S. \& Setayesh K., 2009; Sasao et al., 2010; Hartmann et al., 2003; Noguchi et al., 2008). On the other hand, gliomas have a BBB that is impaired but not absent and this could be reflected in the perfusion data. The difference between these perfusion patterns can contribute significantly to the discrimination of tumor types in cases of peripherally located enhancing tumors when the differential diagnosis includes meningioma and peripheral GBM, and in periventricular enhancing lesions when the differential diagnosis includes choroid plexus papillocarcinoma and GBM (Kaur et al., 2004). It has been reported that MR perfusion is more accurate in determining the true anatomical extent of a lesion as compared to conventional imaging. This is because the perilesional edema often contains microscopic tumors, which manifest on perfusion studies as areas of increased CBV. So perfusion maps may show true tumor margins beyond what is visible on conventional MR imaging. The demonstration of the true anatomical extent of a tumor aids in proper surgical and radiation therapy planning. Perfusion imaging is excellent in the differentiation of tumor recurrence from radiation necrosis, which can be confusing on conventional MR imaging (Hu et al., 2009). On conventional imaging, both recurrent high-grade tumor and radiation necrosis appear as space occupative lesions with post contrast enhancement, perilesional edema, mass effect, and intralesional hemorrhage. Furthermore, for reason not well understood, radiation necrosis most commonly occurs in the tumor bed, even in cases of whole brain irradiation. However, radiation therapy leads to endarteritis and therefore the lesions of radiation necrosis are hypoperfused, whereas, recurrent tumor being most often high-grade, is hyperperfused. Finally, it is expected, that in the future, perfusion imaging may be a surrogate marker to study response in clinical trials of newer antiangiogenic pharmaceuticals. 


\section{Conclusion}

Recent Neuroimaging technology has developed from a purely morphology-based discipline to the complete anatomy, physiology, pathophysiology, hemodynamics and biochemical assessment of a brain tumor. These techniques have important implications in planning therapy and in prognostication. Imaging diagnosis has come from general level deeply into the cellular, molecular, and genetic level. With the advent of the new contrast agents (including organization, organ specific contrast agents, specific gene expression, specific metabolic process, special physiological function contrast agents, etc.), diagnosis and differential diagnosis of specificity will be stronger. Neurosurgeons can design surgery scheme and decide approach for surgical total resection of the tumor during the operation by using a variety of image fusion technology with the application of computer simulation technology and digital image navigation system, which will make the scope of operation more precise and effective, and maximally reserve postoperative neurological function. Recent advances in imaging methods will help us to better assess treatment response, more accurately determine tumor activity during therapy, and differentiate recurrent tumor and treatment related complications.

\section{References}

Amiez C, Kostopoulos P, Champed AS, et al. (2008). Preoperative functional magnetic resonance imaging assessment of higher-order cognitive function in patients undergoing surgery for brain tumors. J Neurosurg, Vol.108, No.2, pp.258-268.

Buascher IM, Skagerberg G, Geijer B, et a1. (2000). Proton MR spectroscopy and preoperative diagnostic accuracy: an evaluation of intracranial mass lesions characterized by stereotactic biopsy findings.Am J Neuroradiol, Vol.21, No.1, pp.84-93.

Caroli E, Orlando ER \& Mastronardi L, et al (2006). Meningiomas infiltrating the superior sagittal sinus: surgical considerations of 328 cases. Neurosurg Rev, Vol. 29, No. 3, pp. 236-41.

Craen R.A., et a1. (2000). A CT method to measure hemodynamics In braintumors: validation and application of cerebral blood flow maps. Am J Neuroradiol, Vol.1, No.3, pp.462-470.

Engelhard $\mathrm{HH}(2001)$. Progress in the diagnosis and treatment of patients with meningiomas. Part I: diagnostic imaging, preoperative embolization. Surg Neurol, Vol.55, No.2, pp.89-101.

Ercan N, Guhekin S, Celik H, et a1. (2004). Diagnostic value of contrast-enhanced fluidattenuated inversion recovery MR imaging of intracranial metastases. American Journal of Neuroradiology, Vol.25, No.5, pp.761-765.

Fountas KN, Kapsalaki EZ, Gotsis SD, et a1.In vivo proton magnetic resonance spectroscopy of brain tumors. Stereotact Funct Neurosurg, Vol.74, No.1, (2000), pp.83-94.

Gerstner ER, Sorensen AG, Jain RK, et al. (2008). Advances in neuroimaging techniques for the evaluation of tumor growth, vascular permeability, and angiogenesis in gliomas. Curr Opin Neurol, Vol.21, No.6, pp.728-735.

Goebell E, Paustenbach S, Vaeterlein O, et al. (2006). Low-grade and anaplastic gliomas: differences in architecture evaluated with diffusion-tensor MR imaging. Radiology, Vol.239, No.1, pp.217-222. 
Gruber S, Stadlbauer A, Mlynarik V, et a1. Proton magnetic resonance spectroscopic imaging in brain tumor diagnosis. Neurosurg Clin N Am, Vol.16, No.1, (2005), pp.101-104.

Guo AC,Cummings TJ, Dash RC, et al. (2002). Lymphomas and high-grade astrocytomas: comparison of water diffusibility and histologic characteristics. Radiology, Vol.224, No.7, pp.177-183.

Haberg A, Kvistad KA, Unsgard G, et al. (2004). Preoperative blood oxygen level-dependent functional magnetic resonance imaging in patients with primary brain tumors: clinical application and outcome. Neurosurgery, Vol.54, No.7, pp.902-914.

Hall WA, Kim P, Truwit CL. (2009). Functional magnetic resonance imaging guided brain tumor resection. Top Magn Reson Imaging, Vol.19, No.4, pp.205-212.

Hamstra DA, Lee KC, Tychewicz JM, et al. (2004). The use of 19F spectroscopy and diffusion weighted MRI to evaluate difference in gene dependent enzyme prodrug therapies. Mol Ther, Vol.10, No.5, pp.916-928.

Hartmann M., Heiland S., Harting I., et al. (2003). Distinguishing of primary cerebral lymphoma from high-grade gliomas with perfusion-weighted magnetic resonance imaging. Neurosci Lett, Vol.338, No.2, pp. 119-122.

Hayashida Y, Hirai T, Morishita S, et al. (2006). Diffusion-weighted imaging of metastatic brain tumors: comparison with histologic type and tumor cellularity. Am J Neuroradiol Vol.27, No.7, pp.1419-1425.

Hemfinghaus S, Pilatus U, Moiler-Hartman W, et a1. Increased choline levels coincide wil1 enhanced proliferative activity of human neuroepithelial brain tumors. NMR Biomed, Vol.15, No.3, (2002), pp.385.

Hirohata M, Abe T, Morimitsu H, et al(2003). Preoperative selective internal carotid artery dural branch embolisation for petroclival meningiomas. Neuroradiology, Vol.45, No.9, pp.656-60.

$\mathrm{Hu}$ L.S., Baxter L.C., Smith K.A., et al. (2009). Relative Cerebral Blood Volume Values to Differentiate High-Grade Glioma Recurrence from Posttreatment Radiation Effect: Direct Correlation between Image-Guided Tissue Histopathology and Localized Dynamic Susceptibility-Weighted Contrast-Enhanced Perfusion MR Imaging Measurements. Am J Neuroradiol, Vol.30, No.3, pp.552-58.

Husstedt HW, Sickert M, Kstler H, et al. (2000). Diagnostic value of the fast-FIAIR sequence in MR imaging of intracranial tumors. Eur Radiol, Vol.10, No.5, pp.745-752 •

Inoue T, Ogasawara K, Beppu T, et al. (2005). Diffusion tensor imaging for preoperative evaluation of tumor grade in gliomas · Clin Neurol Neurosurg, Vol.107, No.3, pp.174-180.

Kaur B., Tan C., Brat D.J., et al. (2004). Genetic and hypoxic regulation of angiogenesis in gliomas. J Neurooncol, Vol.70, No.2, pp.229-43.

Khong PL, Leung LH, Fung AS, et al. (2006). White matter anisotropy in post-treatment childhood cancer survivors: preliminary evidence of association with neurocognitive function. J Clin Oncol, Vol.24, No.6, pp.884-890.

Law M., Cha S., Knopp E.A., et al. (2002). High grade gliomas and solitary metastases: Differentiation by using perfusion and proton spectroscopic MR imaging. Radiology, Vol.222, No.3, pp.715-21. 
Lee HY, Na DG, Song IC, et al. (2008). Diffusion-tensor imaging for glioma grading at 3-T magnetic resonance imaging: analysis of fractional anisotropy and mean diffusivity. J Comput Assist Tomogr, Vol.32, No.2, pp.298-303.

Lu S, Ahn D, Johnson G, et al. (2004). Diffusion-tensor MR imaging of intracranial neoplasia and associated peritumoral edema: introduction of the tumor infiltration index. Radiology, Vol.232, No.1, pp.221-228.

Ludemann L., Warmuth C., Plotkin M., et al. (2009). Brain tumor perfusion: comparison of dynamic contrast enhanced magnetic resonance imaging using T1, T2, and T2* contrast, pulsed arterial spin labeling, and $\mathrm{H} 2 \mathrm{O}$ positron emission tomography. Eur J Radiol, Vol.70, No.3, pp. 465-74.

Manelfe C, Guiraud B \& David J, et al (1973). Embolization by catheterization of intracranial meningiomas. Rev Neurol (Paris), Vol.128, pp. 339-351.

Manelfe C, Lasjaunias P \& Ruscalleda J(1986). Preoperative embolization of intracranial meningiomas. Am J Neuroradiol, Vol.7, No.5, pp.963-72.

Mathews VP, Caldemeyer KS, Lowe MJ, et al. (2000). Brain: gadolinium-enhanced fast fluidattenuated inversion-recovery MR imaging. Radiology, Vol.211, No.1, pp.257-263.

McKnight TR. Proton magnetic resonance spectroscopic evaluation of brain tumor metabolism. Semin Oncol, Vol.31, No.5, (2004), pp.605-617.

Mishra AM, Gupta RK, Jaggi RS, et al. (2004). Role of diffusion-weighted imaging and in vivo proton magnetic resonance spectroscopy in the differential diagnosis of ringenhancing intracranial cystic mass lesions. J Comput Assist Tomogr, Vol.28, No.4, pp.540-547.

Morat BA, Chenevert TL, Lawrence TS, et al. (2005). Functional diffusion map: A noninvasion MRI biomarker for early stratification of clinical brain tumor response. PNAS, Vol.102, No.15, pp.5524-5529.

Murphy PS, Viviers L, Abson C, et a1. Monitoring temozolomide treatment of low -grade glioma with proton magnetic resonance spectroscopy. Br J Cancer, Vol.90, No.6, (2004), pp.781.

Natbavi D.G., Cenic A., Henderson S., et a1. (2001). Perfusion mapping using computed tomography allows accurate prediction of cerebral infarction in experimential brain ischemia. Stroke, Vol.32, No.1, pp. 175-183.

Negendan k W, Sauter R. Proton magnetic resonance spectroscopy in patients with glial tumors : a multicenter study. J Neurosurg, Vol.84, No.3, (1996), pp.449-458.

Noguchi T., Yoshiura T., Hiwatashi A., et al. (2008). Perfusion imaging of brain tumors using arterial spin-labeling: correlation with histopathologic vascular density. Am J Neuroradiol, Vol.29, No.4, pp. 688-93.

Park M.J., Kim H.S., Jahng G.H., et al. (2009). Semiquantitative assessment of intratumoral susceptibility signals using non-contrast-enhanced high-field high-resolution susceptibility-weighted imaging in patients with gliomas: comparison with MR perfusion imaging. Am J Neuroradiol, Vol.30, No.7, pp. 1402-8.

Poupon C, Clark CA, Frouin V, et al. (2000).Regularization of diffusion-based direction maps for the tracking of brain white matter fascicles. Neuroimage, Vol.12, No.8, pp.184-195.

Provenzale JM, McGraw P, Mhatre P, et al. (2004). Peritumoral brain regions in gliomas and meningiomas: investigation with isotropic diffusion weighted $M R$ imaging and diffusion-tensor MR imaging. Neuroradiology, Vol.232, No.6, pp.451-460 • 
Rasmussen IA, Lindseth F, Rygh OM, et al. (2007). Functional neuronavigation combined with intra-operative 3D ultrasound: initial experiences during surgical resections close to eloquent brain areas and future directions in automatic brain shift compensation of preoperative data. Acta Neurochir, Vol.149, No.4, pp.365-378.

Reddy JS, Mishra AM, Behari S, et al. (2006).The role of diffusion-weighted imaging in the differential diagnosis of intracranial cystic mass lesions: a report of 147 lesions. Surg Neurol, Vol.66, No.9, pp.246-250.

Richter HP \& Schachenmayr W(1983). Preoperative embolization of intracranial meningiomas. Neurosurgery, Vol.13, No.3, pp.261-8.

Sasao A. Hirai T. Nishimura S. et al. (2010). Assessment of Vascular Supply of Hypervascular Extra-Axial Brain Tumors with 3T MR Regional Perfusion Imaging. Am J Neuroradiol, Vol.31, No.3, pp.554-58.

Schramm P., Xyda A., Klotz E., et al. (2010). Dynamic CT perfusion imaging of intra-axial brain tumours: differentiation of high-grade gliomas from primary CNS lymphomas. Eur Radiol, Vol.20, No.10, pp.2482-90.

Singh SK, Agris JM, Leeds NE, et a1. (2000). Intracranial leptomeningeal metastases: comparison of depiction at FLAIR and contrast-enhanced MR imaging. Radiology, Vol.217, No.1, pp.50-53

Talos IF, Mian AZ, Zou KH, et al. (2006). Magnetic resonance and the human brain: anatomy, function and metabolism. Cell Mol Life Sci, Vol. 63, No.10, pp.1106-1124.

Toh CH, Castillo M, Wong AM, et al. (2008). Primary cerebral lymphoma and glioblastoma muhiforme: differences in diffusion characteristics evaluated with diffusion tensor imaging. Am J Neuroradiol, Vol.29, No.4, pp.471-475.

Toh CH, Wong AM, Wei KC, et al. (2007). Peritumoral edema of meningiomas and metastatic brain tumors: differences in diffusion characteristics evaluated with diffusion-tensor MR imaging. Neuroradiology, Vol.49, No.6, pp.489-494.

Tomczak RJ, Wunderlich AP, Wang Y, et al. (2000). fMRI for preoperative neurosurgical mapping of motor cortex and language in a clinical setting. J Compur Assist Tomogr, Vol.24, No.6, pp.927-934.

Ulmer JL, Salvan CV, Mueller WM, et al. (2004). The role of diffusion tensor imaging in establishing the proximity of tumor borders to functional brain systems: implications for preoperative risk assessments and postoperative outcomes. Technol Cancer Res Treat, Vol.3, No.6, pp.567-576.

Wang W, Steward CE, Desmond PM. (2009). Difusion tensor imaging in glioblastoma multiforme and brain metastases: the role of $\mathrm{p}, \mathrm{q}, \mathrm{L}$, and fractional anisotropy $\cdot$ Am J Neuroradiol, Vol.30, No.1, pp.203-208.

Wei CW, Guo G, Mikulis DJ. (2007). Tumor effects on cerebral white matter as characterized by diffusion tensor tractography. Can J Neurol Sci, Vol.34, No.1, pp.62-68.

Wetzel SG, Cha S, Law M, et al. (2002). Preoperative assessment of intracranial tumors with perfusion MR and a volumetric interpolated examination: a comparative study with DSA. Am J Neuroradiol, Vol.23, No.11, pp.1767-1774.

Wildfǒrster U, Falk A, Harders A. (1998). Operative approach due to results of functional magnetic resonance imaging in central brain tumors. Comput Aided Surg, Vol.3, No.4, pp.162-165. 
Wintermark M., Philippe J. \& Nabavi T.(2001). Simultaneous Measurement of Regional Cerebral Blod Flow by Perfusion and Stable Xenon CT: A Validation Study. Am J Neuroradiol, Vol.22, No.5, pp.905-914.

Wong ET, Jackson EF, Hess KR, et al. (1998). Correlation between dynamic MRI and outcome in patients with malignant gliomas. Neurology, Vol.50, No.3, pp.777-781.

Wu JS, Zhou LF, Tang WJ, et al. (2007). Clinical evaluation and follow-up outcome of diffusion tensor imaging-based functional neuronavigation: a prospective, controlled study in patients with gliomas involving pyramidal tracts. Neurosurgery, Vol.61, No.5, pp.935-948.

Young G.S. \& Setayesh K. (2009). Spin-Echo Echo-Planar Perfusion MR Imaging in the Differential Diagnosis of Solitary Enhancing Brain Lesions: Distinguishing Solitary Metastases from Primary Glioma. Am J Neuroradiol, Vol.30, No.3, pp.575-7.

Zimmerman RA, Gibby WA, Carmody RF. (1999). Neuroimaging: clinical and physical principles. Springer, ISBN 0387949631, New York • 


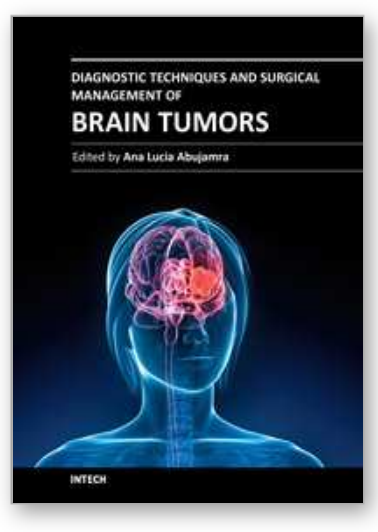

\author{
Diagnostic Techniques and Surgical Management of Brain Tumors \\ Edited by Dr. Ana Lucia Abujamra
}

ISBN 978-953-307-589-1

Hard cover, 544 pages

Publisher InTech

Published online 22, September, 2011

Published in print edition September, 2011

The focus of the book Diagnostic Techniques and Surgical Management of Brain Tumors is on describing the established and newly-arising techniques to diagnose central nervous system tumors, with a special focus on neuroimaging, followed by a discussion on the neurosurgical guidelines and techniques to manage and treat this disease. Each chapter in the Diagnostic Techniques and Surgical Management of Brain Tumors is authored by international experts with extensive experience in the areas covered.

\title{
How to reference
}

In order to correctly reference this scholarly work, feel free to copy and paste the following:

Mingkun Yu, Wei Ye, Xiangqian Qi, Jun Qian, Zhenyang Liu and Wenfeng Fang (2011). Imaging Techniques in Brain Tumor, Diagnostic Techniques and Surgical Management of Brain Tumors, Dr. Ana Lucia Abujamra (Ed.), ISBN: 978-953-307-589-1, InTech, Available from: http://www.intechopen.com/books/diagnostictechniques-and-surgical-management-of-brain-tumors/imaging-techniques-in-brain-tumor

\section{INTECH}

open science | open minds

\section{InTech Europe}

University Campus STeP Ri

Slavka Krautzeka 83/A

51000 Rijeka, Croatia

Phone: +385 (51) 770447

Fax: +385 (51) 686166

www.intechopen.com

\section{InTech China}

Unit 405, Office Block, Hotel Equatorial Shanghai

No.65, Yan An Road (West), Shanghai, 200040, China 中国上海市延安西路65号上海国际贵都大饭店办公楼 405 单元

Phone: +86-21-62489820

Fax: $+86-21-62489821$ 
(C) 2011 The Author(s). Licensee IntechOpen. This chapter is distributed under the terms of the Creative Commons Attribution-NonCommercialShareAlike-3.0 License, which permits use, distribution and reproduction for non-commercial purposes, provided the original is properly cited and derivative works building on this content are distributed under the same license. 